\title{
Strain-specific transmission in an outbreak of ESBL-producing Enterobacteriaceae in the hemato-oncology care unit: a cohort study
}

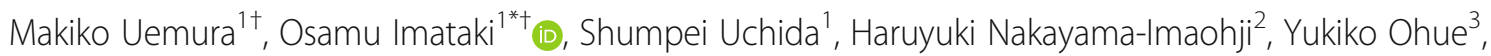
Harumi Matsuka ${ }^{3}$, Hatsune Mori ${ }^{3}$, Hiroaki Dobashi ${ }^{1}$, Tomomi Kuwahara ${ }^{2}$ and Norimitsu Kadowaki ${ }^{1}$

\begin{abstract}
Background: Extended-spectrum $\beta$-lactamase (ESBL)-producing bacteria are resistant to several types of antibiotics excluding carbapenems. A transmissibility of ESBL-producing Enterobacteriaceae would be depending on each bacterial property, however, that has not been elucidated in clinical setting. In this study, we attempted to identify the source of an outbreak of ESBL-producing bacteria in a medical oncology and immunology care unit.

Methods: An ESBL-producing Enterobacteriaceae (ESBL-E) outbreak observed between July 2012 and August 2012 in Kagawa University Hospital was surveyed using various molecular microbiology techniques. We used Pulsed-field gel electrophoresis (PFGE), PCR-based ESBL gene typing, and direct sequence of ESBL gene as molecular microbiology typing method to distinguish each strain.

Results: The typical prevalence of ESBL-E isolation in the unit was 7.0 per month (1.7 per week). The prevalence of ESBL-E isolation during the target research period was 20.0 per month $(5.0$ per week). In total, 19 isolates ( $11 \mathrm{~K}$. pneumoniae and 8 E. coli) were obtained from clinical samples, including four control strains (two each of both bacteria), that were physically different from those obtained from other inpatient units in our hospital. Pulsedfield gel electrophoresis (PFGE) for K. pneumoniae (digested by Xbal) produced similar patterns excluding one control strain. PCR classification of the ESBL gene for $K$. pneumoniae revealed that all strains other than the control strain carried SHV and CTX-M-9. This result was reconfirmed by direct DNA sequencing. Although the outbreak of K. pneumoniae was considered to be "clonal," PFGE and PCR classification of the ESBL genes for E. coli uncovered at least six different "non-clonal" strains possessing individual ESBL gene patterns. According to the result of an antibiogram, the pattern of antimicrobial susceptibility was more variable for K. pneumoniae than for E. coli.

Conclusions: Typing by PFGE and ESBL gene PCR analysis is practical for discriminating various organisms. In our cohort, two outbreaks were concomitantly spread with different transmission strategies, namely clonal and non-clonal, in the same unit. This might represent clinical evidence that transmissibility differs according to the type of strain. We speculated that patient-to-patient transmission of ESBL-E occurred according to the properties of each individual strain.
\end{abstract}

Keywords: Extended-spectrum $\beta$-lactamase (ESBL), Klebsiella pneumoniae, Escherichia coli, Non-clonal outbreak, Multidrug resistance

\footnotetext{
* Correspondence: oima@med.kagawa-u.ac.jp

${ }^{\dagger}$ Equal contributors

${ }^{1}$ Division of Hematology, Rheumatology and Respiratory Medicine,

Department of Internal Medicine, Faculty of Medicine, Kagawa University,

1750-1 Ikenobe, Miki-cho, Kita-gun, Kagawa 761-0793, Japan

Full list of author information is available at the end of the article
}

(c) The Author(s). 2017 Open Access This article is distributed under the terms of the Creative Commons Attribution 4.0 International License (http://creativecommons.org/licenses/by/4.0/), which permits unrestricted use, distribution, and reproduction in any medium, provided you give appropriate credit to the original author(s) and the source, provide a link to the Creative Commons license, and indicate if changes were made. The Creative Commons Public Domain Dedication waiver (http://creativecommons.org/publicdomain/zero/1.0/) applies to the data made available in this article, unless otherwise stated. 


\section{Background}

Extended-spectrum $\beta$-lactamase (ESBL)-producing bacteria are resistant to most beta-lactam antibiotics, including penicillins, cephalosporins, and the monobactam. Consequently, infections caused by ESBL-producing bacteria are difficult to treat. In addition, there are no evidence-based guidelines specifying the infection control for ESBL-producing bacterial outbreak. Therefore, ESBLproducing bacteria easily disseminate and cause nosocomial infection. Infection control of ESBL-producing bacteria is important to prevent outbreaks [1].

An outbreak is defined as the increased incidence of an infectious disease in a specific place during a given period that exceeds the baseline rate for that place and period [2]. According to past reports, outbreaks of ESBL-producing bacteria develop from a single source of infection related to a unique original source, termed "clonal" outbreaks [3]. However, "clonal" outbreak is not the dominant dissemination pattern in limited areas such as care units in healthcare institutes. Global propagation of ESBL genes has been reported among carriers of ESBL-producing bacteria in the community, and it is becoming a general form of bacterial spread [4-6]. In other words, outbreaks of ESBL-producing bacteria can be caused by multiple sources, termed "non-clonal" outbreaks. Opposed to the clonal spread of ESBL-producing bacteria, non-clonal outbreaks are becoming more common [7-9]. In addition to the clonality of outbreak strains, the molecular mechanism of ESBL gene acquisition may contribute to the dynamics of transmission in the clinical setting $[8,10]$. However, differences in the spread of ESBL-producing bacteria have not been fully unveiled.

In our institution, we experienced an outbreak of ESBL-producing bacteria in our medical oncology and immunology care unit. We attempted to identify the original strains of the first index case of infection to clarify the source of infection outbreaks and control nosocomial spread; we performed molecular microbiological analysis of ESBL-E bacteria in our retrospective cohort. The outbreak comprised a mixture of two types of ESBL-producing Enterobacteriaceae (ESBL-E), Klebsiella pneumoniae and Escherichia coli. A main interest of this research is the mechanism of spread of ESBL-E. Our hypothesis is ESBL-producing bacteria has a different transmissibility based on different bacterial properties.

\section{Methods}

\section{Patients and method}

Between July and August 2012, in the medical oncology and immunology care unit of our institute ( 41 beds), 19 strains were obtained from clinical samples during ESBL-E outbreaks. Strains obtained in the unit included the samples of all patients who participated in surveillance during the observed outbreaks. Control strains were prepared from clinically different isolates from heterotopy/heterochrony sampling (labeled as $\odot$ in Figures). We used the first isolate from each clinical sample of all individuals for the analysis. Thus, two or more isolates could be obtained from each individual, such as one strain from stool and the other from blood. Our target species were ESBL-E, namely $K$. pneumoniae and E. coli [8]. Although we observed the outbreak in 2012, the institutional review board approved our research in 2015 .

In this study, we defined two outbreak types according to the source of infection as follows: 1) outbreak from a single source (clonal outbreak) and 2) outbreak from multiple sources (non-clonal outbreak). An outbreak is defined as an increase in the rate of ESBL-E cases or a clustering of new cases in a specific place during a given period. In this report, we defined an unusual increase in ESBL cases as a repeated isolation of ESBL from the medical oncology and immunology care unit, with an incidence $\pm 2 \mathrm{SD}$ over the baseline.

\section{Molecular microbiology methods Pulsed-field gel electrophoresis (PFGE) typing}

Strains isolated from clinical samples were pulsedelectrophoresed on a degraded field agarose gel. The Tenover criteria were used for the separation and identification of each band [2]. Only strains with indistinguishable band profiles were considered to represent the same clone. ESBL-producing $K$. pneumoniae and E. coli isolates were aerobically cultured in brain heart infusion broth (Eiken Chemical Co. Ltd.) for $16 \mathrm{~h}$ at $37^{\circ} \mathrm{C}$. PFGE plugs were prepared using a Gene Path Kit (Bio-Rad) according to the manufacturer's instructions. The plugs were digested overnight with $30 \mathrm{U}$ of $\mathrm{Xba}$ I (New England Biolabs) at $37^{\circ} \mathrm{C}$. The digested DNA bands were separated on $1.0 \%$ agarose gels by PFGE using the CHEFF DR II system (Bio-Rad). PFGE was performed under the following conditions: electric field strength, $6 \mathrm{~V} / \mathrm{cm}$; pulse time, 4$8 \mathrm{~s}$ for $9 \mathrm{~h}$ followed by $8-50 \mathrm{~s}$ for $13 \mathrm{~h}$; and buffer temperature, $14{ }^{\circ} \mathrm{C}$. After electrophoresis, the gels were stained with ethidium bromide $(0.5 \mu \mathrm{g} / \mathrm{ml})$.

\section{Antimicrobial susceptibility testing}

The antibiotic susceptibility of strains isolated from clinical samples was assessed by microdilution methods using an IA20MIC mkII system (Koden Industry Co., Japan). The following antibiotics were used: piperacillin/ tazobactam (PIPC/TAZ), cefotiam (CTM), ceftazidime (CAZ), cefoperazone/sulbactam (CPZ/SBT), cefpirome (CPR), azactam (AZT), minocycline (MINO), imipenem/ cilastatin (IPM/CS), meropenem (MEPM), ciprofloxacin (CPFX), levofloxacin (LVFX), and amikacin (AMK). Breakpoints were adapted according to Clinical and Laboratory 
Standards Institute (CLSI) criteria. Susceptibility was determined by disc diffusion, following the CLSI recommendations for Enterobacteriaceae (CLSI 2010, Performance Standards for Antimicrobial Susceptibility Testing; Twentieth Informational Supplement, M100-S20, Jan. 2010). Drug resistance among the ESBL-producing isolates was assessed by disk dilution methods. Evidence of ESBL production was defined as synergy between co-amoxiclav and at least one of the following antibiotics: cefotaxime (CTX), CAZ, or CPFX. The minimum inhibitory concentrations of CTX and CAZ, with and without clavulanic acid (CVA), were determined subsequently [11].

\section{PCR-based ESBL gene typing}

Molecular ESBL typing was performed as described previously [12]. In detail, ESBL-producing bacteria were screened by PCR/DNA for the detection of $b l a_{\text {TEM }}$, $b l a_{\mathrm{SHV}}$, and $b l a_{\mathrm{CTX}-\mathrm{M}}$. Subsequently, ESBL genes with specific CTX-M subtypes (groups 1, 2, 8 and 9) were characterized using multiplex PCR amplification of the DNA extracted from each strain as previously described [13].

\section{Data collection and ethical issues}

We retrospectively obtained patient data from their medical records. This cohort study was conducted under the approval of the institutional review board (IRB) of our institute (IRB approval No. 27-197).

We numbered the strains as an identifier number which does not compromise patient anonymity.

\section{Results}

The typical prevalence of ESBL-E isolation in the unit was 7.0 per month (1.7 per week). The prevalence of ESBL-E considered positive instances at all the infection sites. A different isolate from the same sampling site in the same individual was not counted double, but a different isolate from the different sampling site in the same individual was counted as a different event. The prevalence of ESBL-E isolation during the target research period (July to August 2012) was 20.0 per month (5.0 per week; Fig. 1 ). We recognized this is an outbreak of ESBL-E in the given period. The time course of ESBL-E isolation in the room is depicted in Fig. 2. Total 19 patients were affected by the outbreak and 15 isolates were used for our analysis. The patients involved in this study were 16 females and 3 males. The median age was 65 years (range $25-73$ years). The patients' diseases and isolation samples were shown in Fig. 2. The median duration from admission to the day of ESBL-E detection was 36 days (range 4-191 days). A cluster outbreak was identified in Room 480, but it was merely a partial phenomenon. The regional outbreak in Room 480 was assumed to be due to transmission. The patients in Room 480 shared a bathroom.

PFGE patterns could not discriminate a series of nine K. pneumoniae strains from clinical samples (with two control strains, K10 and K11; Fig. 3a). However, regarding $E$. coli, all six strains were discriminated as different strains (with 2 control strains, E8 and E9, Fig. 3b). Next we examined the susceptibility of sample strains. In addition, the results of ESBL gene typing were compatible with those of PFGE typing. All ESBL genes extracted from K. Pneumoniae were categorized in CTX-M-9 excluding one control strain (K10) which had carried SMV (Table 1). On another, ESBL genes derived from $E$. coli strains were varied in each strain with TEM, SMV, CTX-M-1, CTX-M-2, or CTX-M-9 (Table 1). The result of an antibiogram were supplied in the supplemental data (Additional file 1: Table S1). The pattern of antimicrobial susceptibility was more variable for $K$. pneumoniae than for E. coli.

In totality, the results of PFGE, antimicrobial susceptibility testing, and ESBL gene typing were not consistent for $K$. pneumoniae and E. coli. All isolates from K. pneumoniae belonged to sequence type (ST) 1308, where control strain (K2) harbored ST1728. This result was consistent with the ESBL gene subtypes (CTX-M-9 identified in K. pneumoniae isolates in our cohort). The outbreak of ST1308 was determined genetically. The antibiogram pattern varied for $K$. pneumoniae. The outbreak of ESBL-producing $K$. pneumoniae could be judged to have the same source of infection in some

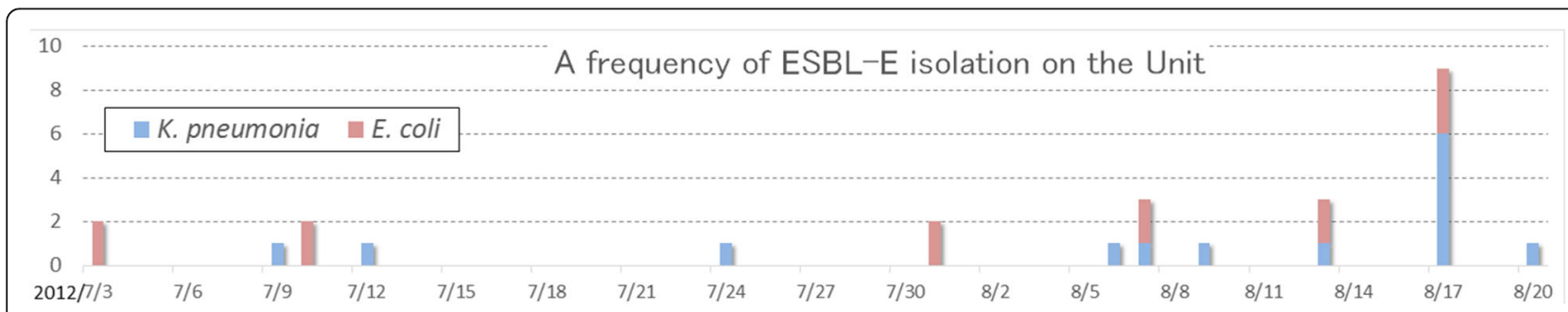

Fig. 1 Frequency of extended-spectrum $\beta$-lactamase-producing Enterobacteriaceae (ESBL-E) isolation in our unit. The incidence of ESBL-E isolation in the microbiological laboratory in our institute was determined. Klebsiella pneumoniae is indicated by the gray bar, and Escherichia coli is denoted by the shadow bar. Only the first microbe isolated from each sample was counted, but the isolation of different specimens in different samples from the same individual was permitted 


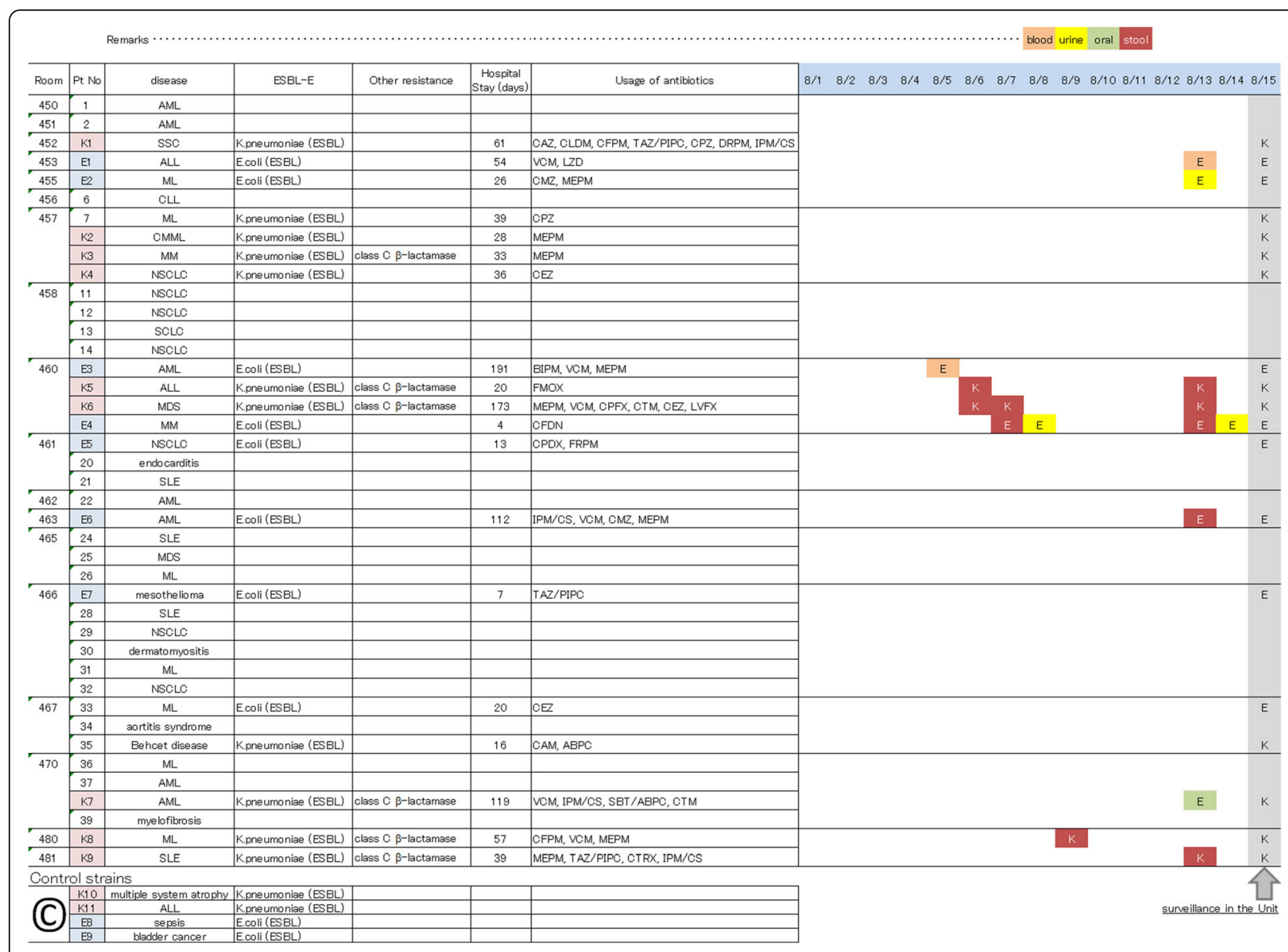

Fig. 2 A description of the patients and the times at which extended-spectrum $\beta$-lactamase-producing Enterobacteriaceae (ESBL-E) were isolated in our unit. Patients' characteristics (age, gender, and diseases) are shown. A unique strain number was provided according to the room number. " $K$ " denotes K. pneumoniae isolates, and " $E$ " indicates E. coli isolates. The types of clinical specimens (blood, urine, oral, and stool) are indicated by red, yellow, green, and brown boxes, respectively. The four control strains, comprising clinically distinct isolates, are shown at the bottom of the list. The rightmost row (row in dark) presents the results of surveillance performed at the end of the outbreak

patients, although not all cases in this episode involved infection with the same strain. For instance, in Room 480 , cluster clonal transmission could have easily occurred. Of note, case $\mathrm{K} 7$ harbored $\mathrm{K}$. pneumoniae and $E$. coli in the oral cavity and stool, respectively. On the other hand, the isolates from $E$. coli were genetically different by PFGE and ESBL gene typing. The outbreak of ESBL-producing $E$. coli strains did not disseminate from the same source of infection as $K$. pneumoniae. Thus, we concluded that a mixed clonal and non-clonal outbreak had occurred in each strain, respectively.

\section{Discussion}

The distinction of strain clonality by antimicrobial susceptibility testing is difficult. Resistance to multiple classes of antibiotics is described as multidrug resistance. Although ESBL exerts a distinctive mechanism for multidrug resistance, subclasses of multidrug resistance have not been elucidated. Thus, our original categorization of drug resistance in this study is not essential, and we considered the antibiogram result to be supplemental data. Conversely, PFGE and ESBL gene type was conducted for ESBL-E strain identification, and we obtained solid results. These analyses of our cohort identified molecular similarity in the PFGE patterns of ESBL-producing $K$. pneumoniae. Although one of the control strains and clinically different strains were not distinguishable, we realized that $K$. pneumoniae strains were of the same origin in the cohort. Identification of the source of infection only based on antimicrobial susceptibility testing in the clinical laboratory would not be practical. Given the dissemination of ESBL-producing bacteria and ESBL gene spread in the community, tracing the source of infection in ESBL-E outbreaks is not beneficial in practice. In any case involving ESBL-producing bacteria, standard precautions should be taken, but tracing the original index case would not contribute to clinical decisions. 


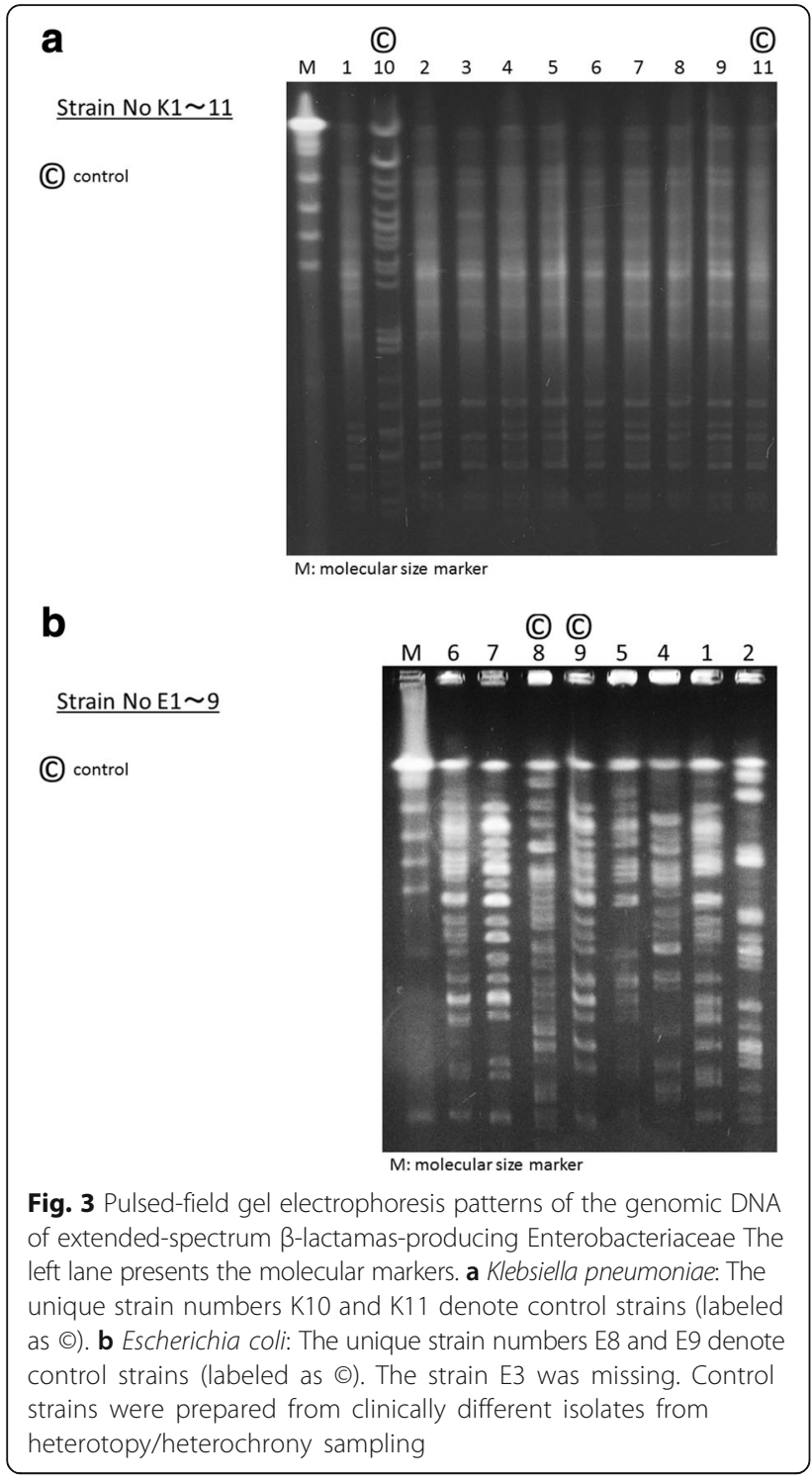

An obvious source of environmental contamination can be easily detected. In our cohort, the clonal outbreak was associated with fecal spread in the bathroom. To estimate the clonality of an outbreak, the location of the outbreak is important. Detecting the ESBL source, i.e., "looking for the culprit," may support in infection-control measures, but it is impossible to achieve complete patient isolation immediately [10]. Patient-to-patient transmission of ESBL-E via the hands of healthcare workers in our unit appeared to be common. Therefore, concomitant outbreaks can occur in a nosocomial setting in which most patients carry or display contamination with ESBL-E. Indeed, our surveillance at the end of the outbreak revealed that $46.3 \%(19 / 41)$ of patients were colonized or infected with ESBL-E (Fig. 2). In multifocal outbreaks, efforts to detect the ESBL source will sometimes end in failure.
Numbers of reports describing clonal outbreak arising from a single source have been published [3-6]. ESBL-E commonly reside in the intestine [10, 14]. Generally, ESBL-E spread from the digestive tract via direct contact, including that with medical items [15]. A hypothesis explaining the unique infection source was not conceivable in our cohort. Yet, in some cases, commonly occurring outbreaks are due to non-clonal expansion. A community-acquired methicillin-resistant Staphylococcus aureus (MRSA) infection is increasing, and the same is for ESBL-E. MRSA, both in the community and hospital settings, colonizes and has low virulence [16]. And ESBL-E can also colonize, and in such cases, it does not become pathogenic in healthy individuals. Some observers in our hospital laboratories, nevertheless, emphasized on food-borne delivery as the source of outbreak merely by referencing a rare report [12]. In accordance with the decision of the infection control team (ICT), they prepared a culture of frozen food in a backward manner to the onset of the outbreak. No ESBL-E bacteria were detected and the ICT ended to conclude communityacquired ESBL-E is a main source of outbreak. Emerging spreads of ESBL-producing bacteria in the community is currently a dominant pattern of infection [4].

In this study, we characterized the different penetrance patterns of the two species of ESBL-E. Our result suggests that these two bacterial species obtained the ESBL gene according to their microbiological properties [1]. PFGE demonstrated that 10 of $11 \mathrm{~K}$. pneumoniae isolates were similar in our analysis. K. pneumoniae isolates in our cohort possessed the CTM-X-9 type of the ESBL gene. bla $a_{\text {СTM-X }}$ has been known to be easily mobilized into plasmids in environmental bacteria [17]. At present, the emergence of community-acquired ESBL-E infections is globally associated with the CTM-X type of ESBL [4], especially with CTM-X-9 in East Asian countries including Japan [18]. CTX-M group ESBL genes are assumed to have more facilitated spread than TEM and SHV group ESBL genes under broad-spectrum antimicrobial selection pressure. Indeed, CTX-M enzymes have rapidly supplanted TEM and SHV group ESBL genes [4]. Some microbiological advantages, which are not yet understood, might possibly contribute to the acquisition of ESBL genes in $K$. pneumoniae rather than in E. coli. On the contrary, in E. coli, ESBL genes are acquired through vector-transporting bacteriophages. Among our cases, E. coli possessed various ESBL genes categorized as TEM, SHV, and CTX-M types.

The digestive tract is the main reservoir for the colonizers of community-acquired ESBL-E, especially E. coli [15]. The probable mechanism of this variety of ESBL genes is intraluminal transmission of resistance genes in the individual gut. In our cohort, surveillance during the outbreak identified $E$. coli among the colonized patients, 
Table 1 Typing of the extended-spectrum $\beta$-lactamase gene

\begin{tabular}{|c|c|c|c|c|c|c|c|c|}
\hline$a$ & & & & & & & & \\
\hline Strain ID & Patient ID & Species & TEM & SHV & CTX-M-1 & CTX-M-2 & CTX-M-8 & CTX-M-9 \\
\hline K1 & 17868 & Klebsiella pneumoniae (ESBL) & & & & & & $\circ$ \\
\hline K10 & 2439551 & Klebsiella pneumoniae (ESBL) & & $\circ$ & & & & $\circ$ \\
\hline K2 & 2607183 & Klebsiella pneumoniae (ESBL) & & & & & & $\circ$ \\
\hline K3 & 1001542 & Klebsiella pneumoniae (ESBL) & & & & & & $\circ$ \\
\hline K5 & 3082844 & Klebsiella pneumoniae (ESBL) & & & & & & $\circ$ \\
\hline K6 & 3083200 & Klebsiella pneumoniae (ESBL) & & & & & & $\circ$ \\
\hline K7 & 2014171 & Klebsiella pneumoniae (ESBL) & & & & & & $\circ$ \\
\hline K7 & 3052387 & Klebsiella pneumoniae (ESBL) & & & & & & $\circ$ \\
\hline K8 & 3073729 & Klebsiella pneumoniae (ESBL) & & & & & & $\circ$ \\
\hline K9 & 1824592 & Klebsiella pneumoniae (ESBL) & & & & & & $\circ$ \\
\hline K11 & 932245 & Klebsiella pneumoniae (ESBL) & & & & & & $\circ$ \\
\hline b & & & & & & & & \\
\hline Strain ID & Patient ID & Species & TEM & SHV & CTM-1 & CTM-2 & CTM-8 & CTM-9 \\
\hline E1 & 2021190 & Escherichia coli (ESBL) & & & & & & $\circ$ \\
\hline E2 & 2535581 & Escherichia coli (ESBL) & & & & & & $\circ$ \\
\hline E3 & 1731148 & Escherichia coli (ESBL) & $\circ$ & & & $\circ$ & & \\
\hline E4 & 2536875 & Escherichia coli (ESBL) & $\circ$ & & & & & $\circ$ \\
\hline E5 & 2304303 & Escherichia coli (ESBL) & & & & & & $\circ$ \\
\hline E6 & 3065667 & Escherichia coli (ESBL) & & & & & & $\circ$ \\
\hline E7 & 2356293 & Escherichia coli (ESBL) & & & & & & $\circ$ \\
\hline E8 & 2362963 & Escherichia coli (ESBL) & $\circ$ & & & $\circ$ & & \\
\hline E9 & 2568787 & Escherichia coli (ESBL) & $\circ$ & & $\circ$ & & & \\
\hline
\end{tabular}

Multiplex PCR results for TEM, SMV, CTX-M-1, CTX-M-2, CTX-M-8, and CTX-M-9 are presented. a) Klebsiella pneumoniae: All strains carried CTX-M-9, excluding one control strain (K10) that carried SMV. b) Escherichia coli: Excluding E1, E2, E5, E6, and E7, all strains carried different types of ESBL genes. The strain identifier numbers presented in the table were not linked to patient's privacy and cannot compromise patient anonymity

providing evidence of fecal carriage. Our results proved that the $E$. coli strains had genetically variable ESBL genes. Thereby, the E. coli outbreak was presumed to be derived from originally community-acquired strains. In general, ESBL genes are mainly transferred in a plasmidmediated manner. ESBL genes have been reported most frequently in K. pneumoniae and E. coli [19]. Our speculation is that the ability to acquire ESBL genes differs among bacteria, and the penetrance of resistance is strain-dependent. Specifically, ESBL-E outbreaks can occur concurrently, as observed in this study. Subsequently, some non-clonal outbreaks can occur coincidentally by the non-clonal strains derived from the community colonizers. Back to the principle of infection control, upon the hypothesis that patient populations in which isolates are genetically similar most likely acquired the organism via patient-to-patient transmission, standard precaution and containment procedures should be enforced irrespective of whether the outbreak is clonal or non-clonal.

Our study has some limitations. First, this is a singleinstitution observation study, and the sample size is small.
Hence, our observation may not represent a universal occurrence. Therefore, other similar clinical data should be accumulated. We were exclusively interested in the difference in the penetrance of the ESBL gene during an outbreak in the clinical setting. The clonal or non-clonal spread of ESBL-E is attributable to microorganisms and individual types of ESBL genes. Second, our study included isolates from both infected and colonized subjects. This protocol might confound the study conclusion. In this study, we investigated the identification of infection source including colonizing strains, in order to prevent an outbreak when the outbreak is spreading and initial action is required immediately. Third, the last limitation of the study concerns the comprehension of the control strains. We selected some independent strains isolated in other wards at different times as control samples for PFGE typing. However, one of the control strains could not be discriminated from other $K$. pneumoniae strains. Despite some environmental contamination, there was no evidence of transmission from patients known to be colonized or infected with ESBL-producing $K$. pneumoniae and E. coli who were discharged from the hospital or admitted to other wards. 


\section{Conclusions}

We observed that clonal and non-clonal outbreak of ESBL-E corresponding to the difference of bacteria. We were unable to identify the common source of the outbreak among the two organisms, i.e., K. pneumoniae and E. coli, in the cohort. These two organisms would possibly transmit ESBL genes according to each bacteria's biological ability. This molecular microbiological difference did not appear in the mapping of ESBL-E spread, but it did reflect organism-specific outbreaks whether clonal or non-clonal. Our findings provide an outlook of the concurrent expansion of clonal and non-clonal outbreaks. Our cohort represents an endeavor to detect the index case of the outbreak and clarify that a subject harboring ESBL-E is no longer essential in the era of widespread ESBL-E dissemination in the community. Thus far, we confirmed the importance of substantial standard precautions in the background of the expansion of multidrug-resistant organisms in the community.

\section{Additional file}

Additional file 1: Table S1. Antibiogram of extended-spectrum $\beta$ lactamase-producing Enterobacteriaceae. The susceptibility of each strain for the given antibiotics is presented in the table as the susceptibility titer number. The number are shown as minimum inhibitory concentration (MIC) $\mu \mathrm{g} / \mathrm{mL}$. Resistance is indicated by bold characters. Different clones from the same specimen in the same individual were numbered using subnumbers, e.g., XX_1 and XX_2. We classified the clones into resistance groups as follows: Group A, sensitive to all antibiotics excluding cephalosporins (e.g., CAZ); group B, resistant to PIPC and cephalosporins; group C, resistant to quinolones and cephalosporins; and group D, resistant to all antibiotics excluding carbapenems. Based on our original grouping criteria, the strains could be categorized to four resistance groups. We categorized the strains in the four groups and attempted to comprehend the clonality of each bacterium. (a) Klebsiella pneumoniae: K3, K4, K8, and $\mathrm{K} 11$ were classified into group $\mathrm{A} ; \mathrm{K} 5, \mathrm{~K} 7$, and $\mathrm{K} 9$ into group $\mathrm{B} ; \mathrm{K} 2$ and K10 into group C; and K6 into group D. (b) Escherichia coli: E2 was categorized in group $\mathrm{A}$, and the other strains were categorized in group D. Thus, the pattern of antimicrobial susceptibility was more variable for $K$. pneumoniae than for E. coli. (The control strains are remarked as (C).). The strain identifier numbers presented in the table were not linked to patient's privacy and cannot compromise patient anonymity. (DOCX $19 \mathrm{~kb}$ )

\section{Acknowledgments}

The authors have no conflicts of interest to disclose. We have none funding source.

\section{Funding}

None. This study was supported by internal funding.

\section{Availability of data and materials}

All data are shown in the manuscript. The original data are available.

\section{Authors' contributions}

$\mathrm{OI}$ and MU managed the patient's case and wrote the manuscript. SU contributed to the literature search. $\mathrm{HNI}$ and TK performed the microbiological assay and interpreted the result. YO, HM, and HM managed the data and suggested important intellectual content. HD reviewed the manuscript. TK made substantial contributions to the concept and design of this report. OI and TK organized and managed this work. All authors read and approved the final version of the manuscript.

\section{Competing interests}

The authors have no conflicts of interest to disclose.

\section{Consent for publication}

Not applicable.

\section{Ethics approval and consent to participate}

We retrospectively obtained patient data from their medical records. This retrospective research was conducted under the approval of the institutional review board (IRB) of our institute, Kagawa University, Faculty of Medicine IRB (IRB approval No. 25-005).

\section{Transparency declarations}

None to declare.

\section{Author details}

'Division of Hematology, Rheumatology and Respiratory Medicine, Department of Internal Medicine, Faculty of Medicine, Kagawa University, 1750-1 Ikenobe, Miki-cho, Kita-gun, Kagawa 761-0793, Japan. ²Division of Molecular Microbiology, Kagawa University, Kagawa, Japan. ${ }^{3}$ Nursing Division, Kagawa University Hospital, Kagawa, Japan.

Received: 3 September 2016 Accepted: 20 December 2016 Published online: 05 January 2017

\section{References}

1. Turner PJ. Extended-spectrum beta-lactamases. Clin Infect Dis. 2005;41 Suppl 4:S273-5.

2. Tenover FC, Arbeit RD, Goering RV, et al. Interpreting chromosomal DNA restriction patterns produced by pulsed-field gel electrophoresis: criteria for bacterial strain typing. J Clin Microbiol. 1995;33:2233-9.

3. Paterson DL, Yu VL. Extended-spectrum beta-lactamases: a call for improved detection and control. Clin Infect Dis. 1999;29:1419-22.

4. Woerther PL, Burdet C, Chachaty E, et al. Trends in human fecal carriage of extended-spectrum $\beta$-lactamases in the community: toward the globalization of CTX-M. Clin Microbiol Rev. 2013;26:744-58.

5. Peirano G, Pitout JD. Molecular epidemiology of Escherichia coli producing CTX-M beta-lactamases: the worldwide emergence of clone ST131 O25:H4. Int J Antimicrob Agents. 2010;35:316-21.

6. Coque TM, Baquero F, Canton R. Increasing prevalence of ESBL-producing Enterobacteriaceae in Europe. Euro Surveill. 2008;13(47).

7. Goddard S, Muller MP. The efficacy of infection control interventions in reducing the incidence of extended-spectrum $\beta$-lactamase-producing Enterobacteriaceae in the nonoutbreak setting: A systematic review. Am J Infect Control. 2011;39:599-601.

8. Falagas ME, Karageorgopoulos DE. Extended-spectrum beta-lactamaseproducing organisms. J Hosp Infect. 2009;73:345-54.

9. Manzur A, Tubau F, Pujol M, et al. Nosocomial outbreak due to extendedspectrum-beta-lactamase- producing Enterobacter cloacae in a cardiothoracic intensive care unit. J Clin Microbiol. 2007:45:2365-9.

10. Pfaller MA, Segreti J. Overview of the epidemiological profile and laboratory detection of extended-spectrum beta-lactamases. Clin Infect Dis. 2006; 42 Suppl 4:S153-63.

11. Pitout JD, Nordmann P, Laupland KB, Poirel L. Emergence of Enterobacteriaceae producing extended-spectrum beta-lactamases (ESBLs) in the community. J Antimicrob Chemother. 2005;56:52-9.

12. Calbo E, Freixas N, Xercavins $M$, et al. Foodborne nosocomial outbreak of SHV1 and CTX-M-15-producing Klebsiella pneumoniae: epidemiology and control. Clin Infect Dis. 2011:52:743-9.

13. Viau RA, Hujer AM, Marshall SH, et al. "Silent" dissemination of Klebsiella pneumoniae isolates bearing K. pneumoniae carbapenemase in a long-term care facility for children and young adults in Northeast Ohio. Clin Infect Dis. 2012;54:1314-21.

14. Karanika S, Karantanos T, Arvanitis M, Grigoras C, Mylonakis E. Fecal Colonization With Extended-spectrum Beta-lactamase-Producing Enterobacteriaceae and Risk Factors Among Healthy Individuals: A Systematic Review and Metaanalysis. Clin Infect Dis. 2016;63:310-8.

15. Johnson JR, Russo TA. Extraintestinal pathogenic Escherichia coli: "The other bad E. coli.". J Lab Clin Med. 2002;139:155-62.

16. Safdar N, Maki DG. The commonality of risk factors for nosocomial colonization and infection with antimicrobial-resistant Staphylococcus aureus, enterococcus, 
gram-negative bacilli, Clostridium difficile, and Candida. Ann Intern Med. 2002;136:834-44.

17. Cantón R, González-Alba JM, Galán JC. CTX-M Enzymes: Origin and Diffusion. Front Microbiol. 2012:3:110.

18. Chong Y, Shimoda S, Yakushiji H, et al. Community spread of extendedspectrum $\beta$-lactamase-producing Escherichia coli, Klebsiella pneumoniae and Proteus mirabilis: a long-term study in Japan. J Med Microbiol. 2013;62:1038-43.

19. Bush K, Jacoby GA, Medeiros AA. A functional classification scheme for beta-lactamases and its correlation with molecular structure. Antimicrob Agents Chemother. 1995;39:1211-33.

Submit your next manuscript to BioMed Central and we will help you at every step:

- We accept pre-submission inquiries

- Our selector tool helps you to find the most relevant journal

- We provide round the clock customer support

- Convenient online submission

- Thorough peer review

- Inclusion in PubMed and all major indexing services

- Maximum visibility for your research

Submit your manuscript at www.biomedcentral.com/submit
Biomed Central 